\title{
Prosocial Decision Making in an Operant Box Paradigm Promotes Visual Communication and Complex Behavioral Sequences in Adolescent Rat Dyads
}

\author{
Valérie Charron†, Joey Talbot', and Hélène Plamondon* \\ Behavioural Neuroscience Group, School of Psychology, University of Ottawa \\ 'Both researchers contributed equally to this research \\ *Corresponding author (Email: Helene.Plamondon@uottawa.ca) \\ Citation - Charron, V., Talbot, J., \& Plamondon, H. (2022). Prosocial decision making in an operant box paradigm \\ promotes visual communication and complex behavioral sequences in adolescent rat dyads. Animal Behavior and \\ Cognition, 9(1), 53-71. https://doi.org/10.26451/abc.09.01.05.2022
}

\begin{abstract}
The adolescence period is marked by intense social play behavior in rats, shown to influence social, cognitive, and emotional processes. The goal of this study was to assess the ability of adolescent rats to display prosocial behaviors through a sharing task and to learn prosociality from vicarious observation. The paradigm involved a pretraining phase, using a two-chamber operant box with two reward differentiated levers on the actor side, providing one and two sucrose pellets respectively upon pressing. Dyads where actors were not exposed to pretraining session acted as controls. The prosocial phase ensued, where an easy lever pressed dispensed one pellet while a hard lever pressed dispensed one reward to both the actor and observer in the adjacent chamber. Actor and observer rats then switched roles enabling vicarious learning assessment. Findings revealed pretraining to be critical for behavior and task contingency in adolescent rats. Complex behavioral sequences marked by increased visual communication between dyads was observed. Despite the diversity of behaviors, observer rats failed to learn prosocial behaviors. This study shows pretraining to act as a key element promoting behavioral interactions; the thorough behavioral analysis performed highlights the ability for adolescent rats to display a richness of behaviors when paired with a congener. Another interesting finding was the ability for rats to learn prosocial behaviors, but the inability to learn such behaviors by observation. These findings call for further studies to understand prosocial behaviors in rodents and their ability to learn such behaviors from a congener.
\end{abstract}

Keywords - Social behavior, Pretraining, Operant conditioning, Adolescence, Rat

Research on prosocial behaviors in rats is growing within the fields of comparative psychology and social neuroscience. The concept of prosocial behavior in rodents is commonly referred to as any helping behaviors towards a congener to improve their well-being (e.g., freeing a restrained congener, sharing a food reward; Ben-Ami Bartal et al., 2014, Cronin, 2012; Hernandez-Lallement et al., 2015). Many factors can influence the likelihood of rodents behaving in a prosocial manner, such as developmental stages, strain, the paradigm used and the training components (Meyza et al., 2017). Although the use of aversive tasks (i.e., water aversive task, fear conditioning paradigms) has initially predominated in studies assessing prosocial behaviors in rodents (Church, 1959; Greene, 1969; Lee et al., 2018; Meyza et al., 2017; Sato et al., 2015; Yusufishaq \& Rosenkranz, 2013), the last decade has seen an emergence of paradigms using sharing or freeing tasks (Sivaselvachandran et al., 2018). In this context, the study conducted by Ben-Ami Bartal and colleagues (2011) represents a hallmark. These authors were the first to show a rat's motivation to free a cagemate from a restrainer, even when no physical contact was possible once the rat was free. 
Moreover, in around half of situations where a choice between freeing the cagemate or receiving palatable chocolate chips had to be made, rats decided to first free the congener and share the food reward (Ben-Ami Bartal et al., 2011). In the same vein, Hernandez-Lallement and colleagues (2015) used a double T-Maze to conceptualize a prosocial choice task in which rats could make a choice between attribution of a single or a mutual food reward by entering a specific maze compartment (i.e., the own-reward compartment, or the both-reward compartment). Rats chose the both-reward compartment when paired with a partner, but not a rat toy, showing that rats preferred the mutual reward with another conspecific (Hernandez-Lallement et al., 2015).

Vicarious learning is defined as a behavior acquired through the observation of a conspecific's action and its consequences (Schaik et al., 2016; Zentall, 2016). Similar to prosocial models, vicarious learning has mostly been studied using aversive paradigms (Meyza et al., 2017). Recently, research has moved towards models limiting the use of aversive stimuli (e.g., electric shocks). A study by Yamada and Sakurai (2018) proposed a paradigm where a rat placed in the middle of a Barnes' maze observed its congener performing the task (i.e., finding a black box allowing it to escape from aversive light). The observer rats were able to find the box faster and escape more quickly than the rats that had not previously observed a congener (Yamada \& Sakurai, 2018). It is established that rodents can display prosocial-like behaviors and learn different tasks from the observation of a congener (Ben-Ami Bartal et al., 2011; Hernandez-Lallement et al., 2015; Meyza et al., 2017; Yamada \& Sakurai, 2018). However, abilities of rodents to learn prosocial behaviors through vicarious observation remains undetermined.

To date, studies have focused on characterizing prosociality during adulthood, and other developmental periods have been neglected. Considering the well-known and significant brain reorganization taking place during adolescence that is marked by behavioral changes, heightened brain development, and cognitive maturation (Caballero et al., 2016; Walker et al., 2017), it is surprising that few studies have assessed prosocial behavior and associated communication patterns in adolescent rats. Adolescence is a period of remodeling of the brain architecture in which hormones play a role along with experience (Sisk \& Zehr, 2005). The prefrontal cortex, which undergoes a prolonged course of maturation well after puberty, is exquisitely vulnerable in the adolescent period as well as the interconnected amygdala, hippocampus and mesolimbic/nigrostriatal systems (Caballero et al., 2016), all of which might influence prosocial behaviors. Furthermore, the adolescence period is characterized by intense social play behaviors, which is of particular interest when studying prosocial behaviors (Manduca et al., 2014), where rapid growth in learning capacity and adaptability have been described (Dahl et al., 2018). For instance, social play in adolescent rats, which consists of rough-and-tumble play, is known to induce positive vocalization and play an important role in emotional, social and cognitive development (Achterberg et al., 2019; Lampe et al., 2019). Adolescent rats deprived of social interactions show increased anxiety and depressive-like behaviors (Burke et al., 2017), which supports the idea that social contact is crucial for healthy neurobehavioral maturation.

Notably, studies assessing prosocial behaviors have indicated possible benefits of using a pretraining phase to familiarize rats to the environment and the rewards associated with a specific action (e.g., lever press; Oomen et al., 2013). Brady and Floresco (2015) showed that 10 to 20 sessions are sufficient in operant procedures to familiarize rats with the levers and associated rewards. The reward provided during pretraining can vary, some using a fixed ratio of one reward/reinforcement per lever press (Brady \& Floresco, 2015) while others recommend the use of different lever-reward contingencies to facilitate lever discrimination and later test the willingness of the actor to work harder towards reward delivery to a congener in the prosocial phase (Horner et al., 2013). Although pretraining influences learning in operant tasks, its contribution to the development of communication patterns in rat dyads has not been explored. Communication and behavioral interactions can be considered key elements in understanding prosocial behaviors in rat dyads (Martin et al., 2014). These can be studied with ultrasonic vocalizations, which can unravel information about the emotional state of rats such as positive affect (Engelhardt et al., 2018; Łopuch \& Popik, 2011; Seffer et al., 2014) or with statistical tools such as discriminant analyses (O'Connor, 1999). Results from Ben-Ami Bartal and colleagues (2011) highlight the importance of studying behaviors in addition to task performance. Rats that opened the restrainer were considered 
prosocial based on the performed task (i.e., opening of the restrainer) made in conjunction with behavioral interactions observed before (circling the restrainer, contacting the trapped cagemate through the holes in the restrainer; Ben-Ami Bartal et al., 2011). It is paramount to pursue the assessment of behaviors in addition to task performance to better understand prosociality in rodents.

Different rat strains have been tested in prosocial paradigms, including Long-Evans (Atsak et al., 2011; Hernandez-Lallement et al., 2015) and Sprague-Dawley rats (Ben-Ami Bartal et al., 2011; Lee et al., 2018). Although both strains are known to be social and perform well in operant tasks (Ku et al., 2016), studies have suggested behavioral differences between these strains. Pigmented rats (e.g., Long-Evans) are known to perform better in visual and lever press tasks (Andrews et al., 1995; Gökçek-Saraç et al., 2015) and to be more active during daytime than albino rats (e.g., Sprague-Dawley; Stryjek et al., 2013), whereas Sprague-Dawley rats engage more frequently and for longer periods of time with a novel congener during a social dyad task than Long-Evans rats (Ku et al., 2016). Strain-specific responses remain poorly characterized, although such assessments may help establish genomic changes mediating prosocial, biochemical and behavioral responses.

The main objective of this study was to characterize baseline prosocial behaviors in adolescent Long-Evans and Sprague-Dawley male rats using an operant box paradigm. As part of this endeavor, we determined the role of a pretraining phase in shaping the actor rats' prosocial responses (i.e., reward sharing). An additional goal of this study was to assess vicarious learning and determine if being exposed to prosocial behaviors once in the observer role influence behavior upon becoming the actor. Finally, this study is the first to use transitional probabilities analysis to determine the influence of prosocial behavior on communication patterns and behavioral responses (complexity and repertoire of behavioral sequences) observed in adolescent rat dyads.

\section{Method}

\section{Ethics Statement}

All experimental procedures were approved by the University of Ottawa Animal Care Committee (Protocol Review Group) and met the guidelines put forth by the Canadian Council on Animal Care.

\section{Animals}

Male Long-Evans and Sprague-Dawley rats $(n=20)$ were obtained from Charles River Laboratory (Québec, Canada). Rats arrived at the animal care facility at post-natal day (PND) 23 and 28 for the pretrained and naive (not exposed to pretraining) group, respectively (see Figure 1A for group repartition). Considering ethical reasons (e.g., use of a minimal number of animals), assessment of strain differences on prosocial behaviors was limited to the naive group only. Upon arrival, rats of each strain were housed in pairs in a temperature/humidity-controlled room $\left(22^{\circ} \mathrm{C} / 60 \%\right.$ humidity level) under a $12 \mathrm{~h}$ dark/light cycle (lights on at 7 AM) with ad libitum access to food (Teklad Global 18\% Protein Diet manufactured by Envigo) during the 2 days of habituation. Daily handling began on day 3 of arrival. Starting on day 5, rats were food restricted at $85-90 \%$ of free feeding body weight. Rats were regularly weighed, and all showed adequate weight gain as the experiment progressed. Upon testing initiation, Long-Evans and SpragueDawley body weights ranged between 200-225 g and 108-200 g, respectively. Testing of the pretrained and naive rats was initiated on PND 30 and PND 35, respectively. Food rewards used during the testing consisted of $45 \mathrm{mg}$ chocolate flavored sucrose tablets (Test Diet, USA).

\section{Apparatus}

Two Coulbourn Instruments Habitest ${ }^{\mathrm{TM}}$ operant boxes $(30 \mathrm{~cm} \times 25 \mathrm{~cm} \times 30 \mathrm{~cm})$ were modified as to our protocol's needs (see Figure 2). Graphic State 3.03 ${ }^{\mathrm{TM}}$ from Coulbourn Instruments was used as the data acquisition software. 


\section{Figure 1}

\section{Phases of the Experiment}

A)

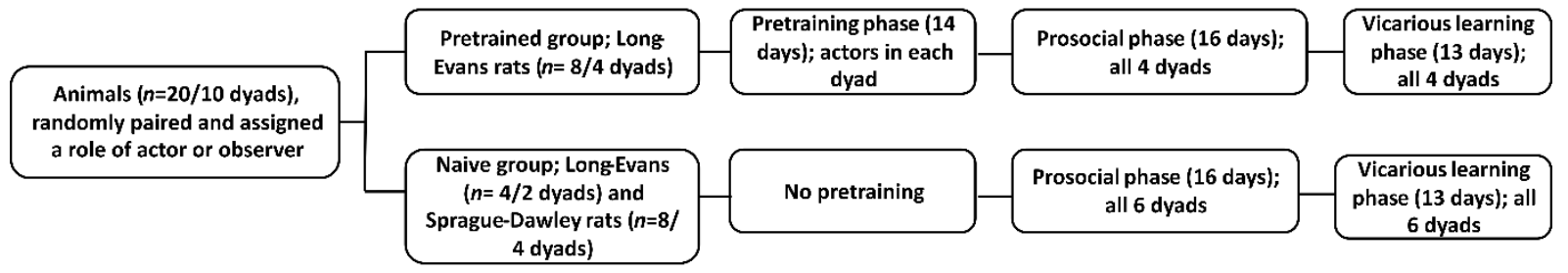

B)

1. Pretraining phase

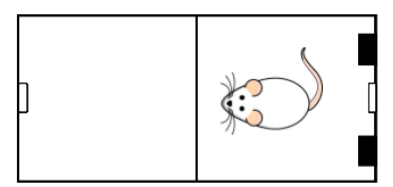

2. Prosocial phase

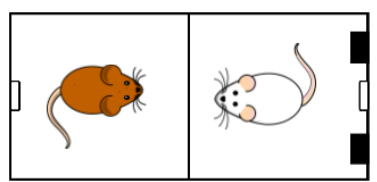

3. Vicarious learning phase

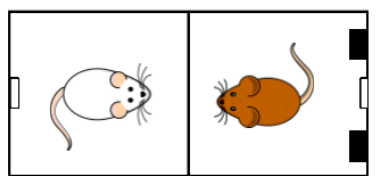

Note. A) The three conditions (pretraining, prosocial and vicarious phases) included testing sessions that lasted 60 min. Testing of the pretrained and naive rats was initiated on PND 30 and PND 35, respectively. B) 1- The pretraining phase enabled an actor rat to learn the reward contingencies associated with the two levers. Actor rats in the naive group were not exposed to this phase. 2The prosocial phase assessed the willingness of an actor rat (right side) to press a hard lever delivering one pellet to itself and one to the congener (prosocial behavior) or select the easy lever delivering one pellet to itself. 3- Following the prosocial phase, actor and observer rats switched compartments enabling the assessment of vicarious learning in naive and pretrained observer rats.

\section{Figure 2}

\section{Modified Coulbourn Operant Box}

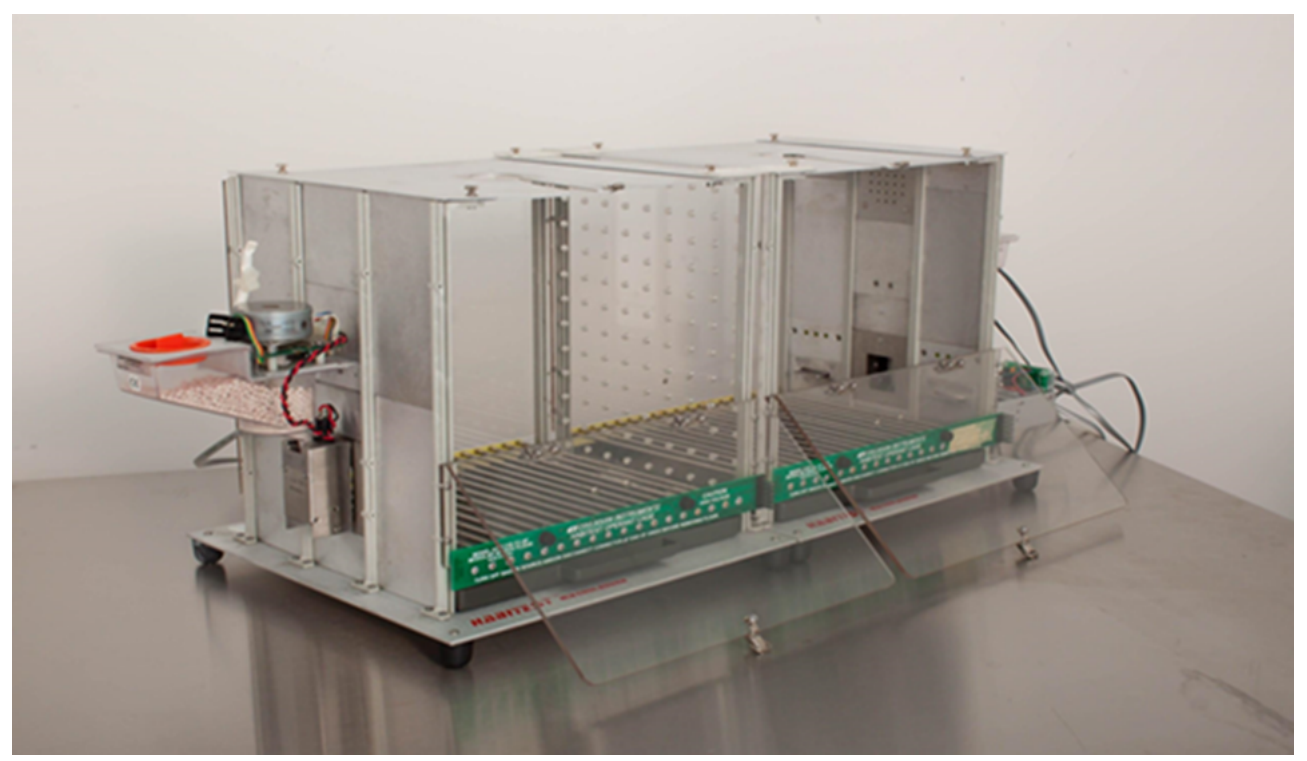

Note. Modified Coulbourn Instruments $\mathbb{R}$ Habitest ${ }^{\mathrm{TM}}$ operant box with two compartments separated by a perforated acrylic sheet. On the right side of the box (the actor side), two levers, cue lights and a feeder were present while on the left side (the observer side), only a feeder was present. 


\section{Procedure}

Before the experiment began, paired rats were randomly assigned the initial role of actor or observer. The pretrained group was composed of eight Long-Evans, for a total of four dyads of rats. The naive group was composed of eight Sprague-Dawley and four Long-Evans, for a total of six dyads of rat (dyads of actor-observer were formed within each strain). For detailed information about the group repartition, see Figure 1A. Rats were brought to an acclimation room $30 \mathrm{~min}$ before testing. The acclimation and testing rooms were isolated, and ceiling lights turned on, maintaining the rat's diurnal rhythm. The testing environment consisted of two annexed operant boxes facing a camera and surrounded by white curtains. Experimenters left the room during the 60 min testing session, minimizing external distractors. All testing sessions lasted $60 \mathrm{~min}$, five consecutive days/week. All sessions were recorded for further behavioral analyses. For visual representation of the tasks, see Figure 1B.

\section{Pretraining Phase}

Only Long-Evans actor rats $(n=4)$ that were part of the pretrained group were exposed to pretraining. Rats were placed in the right-hand side chamber compartment (left side remaining empty) with accessibility to the two levers, the easy lever ( $35 \mathrm{~g}$ of pressure) delivering one pellet and the hard lever ( 75 $\mathrm{g}$ of pressure) delivering two pellets. The hard lever was identified by a flashing cue light placed above it, while the easy lever was identified by a steady light. Levers were changed regularly with their associated light stimuli once every 5 days of testing to prevent location preference. The pretraining phase lasted a total of 14 days, based on the recommendation of Brady and Floresco (2015), who stated that a pretraining phase should last between 10 to 20 days. After the 14-day pretraining period, all rats reached the minimum criterion of 90 presses on each lever per session, confirming that sufficient exposure to the levers had occurred for all rats. Actor rats from the naive group were not exposed to any form of pretraining.

\section{Prosocial Phase}

During this phase, the lever settings remained the same as during the pretraining. All dyads of rats ( $n=10$ dyads) participated in this phase, which lasted 16 days for both groups. The duration of this phase was based on the pretraining and previous studies (Ben-Ami Bartal et al., 2014; Tomek et al., 2019). The prosocial phase introduced an observer rat (the actor's cagemate), which was placed in the compartment adjacent to the actor, with sole access to a feeder. The actor's task remained identical with the exception that a press on the hard lever now provided one pellet to itself and one pellet to the observer, while the easy lever dispensed a pellet to the actor only.

\section{Vicarious Learning Phase}

The vicarious learning phase was meant to assess how well the observer performed after witnessing the prosocial phase. This phase used the same experimental protocol, except that the observer rat exchanged roles with their previous assigned actor rat (the actor is now in the other compartment, witnessing the task). This third phase lasted 13 days for both groups (Bem et al., 2018; Carlier \& Jamon, 2006).

\section{Behavioral Recording}

All sessions were recorded, the frequency and duration of behaviors was coded by two graduate students using the Boris behavioral coding software (Friard \& Gamba, 2016). To assess the intercoder reliability, four sessions (equal to four hours of video) were randomly selected, coded and analyzed. The intercoder reliability for all variables was ranging from .81 to .91, using the time-unit Kappa in the GSEQ 5.1 software, translating to a strong level of agreement (Quera et al., 2007). In total, six behaviors were 
observed and quantified (see Table 1 for behavior description). The behavioral recordings were used to assess and compare the behavior frequency and transition in the actor and observer rats.

Table 1

Operational Definition of Analyzed Behaviors

\begin{tabular}{|c|c|c|}
\hline Behavior & Action & Operational Definition \\
\hline Communication & Mutual & $\begin{array}{l}\text { Both rats stand in contiguity of the acrylic walls, side } \\
\text { by side or facing each other, rearing or standing on } \\
\text { four paws, excluding grooming. }\end{array}$ \\
\hline Looking & Mutual & $\begin{array}{l}\text { Both rats directly looking at each other from a } \\
\text { distance of the acrylic wall. }\end{array}$ \\
\hline Prosocial decision making & Actor & $\begin{array}{l}\text { The actor approaches the easy lever, actively } \\
\text { explores it (i.e., touching with nose or paws, } \\
\text { sniffing, looking), and decides to press the hard } \\
\text { lever. }\end{array}$ \\
\hline Selfish decision making & Actor & $\begin{array}{l}\text { The actor goes towards the hard lever, actively } \\
\text { explores it (i.e., touching with nose or paws, } \\
\text { sniffing, looking), and decides to press the easy } \\
\text { lever. }\end{array}$ \\
\hline *Fetching hard lever reward & Observer & $\begin{array}{l}\text { When the actor presses the hard lever, the observer } \\
\text { goes looking into his feeder. *Only when following } \\
\text { a prosocial decision making. }\end{array}$ \\
\hline *Fetching easy lever reward & Observer & $\begin{array}{l}\text { When the actor presses the easy lever, the observer } \\
\text { goes looking into his feeder. *Only when following } \\
\text { a selfish decision making. }\end{array}$ \\
\hline
\end{tabular}

Note. Behavioral data logging enabled comparison of behavioral responses and characterized behavioral sequences in pretrained and naive rat dyads. *Fetching behaviors enabled the assessment the observers' understanding of the task. A rat that goes towards its own feeder when its congener presses the hard lever could indicate an understanding that a reward pellet will also be delivered to itself. However, a rat that goes towards its feeder when the easy lever was press can indicate that it did not understand the function of levers and thus the task itself.

The discriminant analysis suit developed by O'Connor (1999) produces a series of statistical analyses that allows the identification of behavioral sequences and the comprehension of its asymmetrical distribution. Within the application, there is the transitional probability analysis, which produces a likelihood ratio of behavioral sequences (e.g., There is an "X\%" chance that behavior " $A$ " will be followed by behavior "B") accompanied by Z scores, significance levels, and descriptive statistics. Based on the observed frequencies of the monitored behaviors, this analysis compares real observations with expected behavioral transitions that assumes no interdependency between behaviors. It produces a statistical significance level between behaviors and their transitions indicating if the likelihood ratio is valid and accurate. For additional information on likelihood ratio calculations and transitional probability analysis, see O’Connor (1999).

\section{Statistical Analysis}

All statistical analyses were performed using IBM SPSS (version 23). Data were analyzed using a one-way multivariate analysis of variance (MANOVA) for lever press (easy and hard presses being two separate measures) differences between groups and the frequencies of behaviors. For each of the MANOVA analysis, the mean of each rat was analyzed thus removing the factor of variability. Behavioral sequences between groups were analyzed using transitional probability analysis an alpha level of .05 (O'Connor, 1999). A strain comparison of performance (easy and hard lever presses) and behavior frequencies were assessed using independent samples $t$-tests. Homogeneity of variance was verified using the Levene's test. 
When significant, the Welch $t$ Test Statistic was used. Data are presented as mean \pm SEM. Statistical significance was set to $p<.05$.

\section{Results}

\section{Group Comparisons}

A one-way MANOVA assessed two separate measures of performance: the mean of lever presses on the easy and hard levers. Groups were the following: 1) pretrained actors, 2) naive actors, 3) pretrained observers and 4) naive observers. For the visual representation of the data, see Figure 3A and B.

Preliminary assumption checking revealed that data was normally distributed, as assessed by a Normal Q-Q plot. There was one univariate outlier (removed from the dataset), as assessed by boxplot and eight multivariate outliers assessed by the Mahalanobis distance $(p<.001)$. After analysis with and without the eight outliers, they were kept in the data as the decision rule did not affect the result of the one-way MANOVA. There were linear relationships, as assessed by scatterplot and no multicollinearity between the variables $(r=.272, p<.001)$. There was no homogeneity of variance-covariance matrices, as assessed by Box's M test $(p<.001)$. Because this assumption was not fulfilled, a Pillai's Trace effect was used instead of a Wilks' $\Lambda$. Additionally, since the Levene's test of equality of variances was significant $(p<.05)$, a lower alpha level of $.025(p<.025)$ was used. Considering a small sample size, a power analysis was conducted using G*Power (version 3.1.9.4) for the MANOVA analysis (O'Keefe, 2007). A statistical power of $0.733(\beta=0.733)$ was achieved using conservative alpha value of $.025(\alpha=.025)$.

\section{Figure 3}

Pretraining Modulates Prosocial Choices but Does Not Affect Vicarious Learning

A) Prosocial Phase

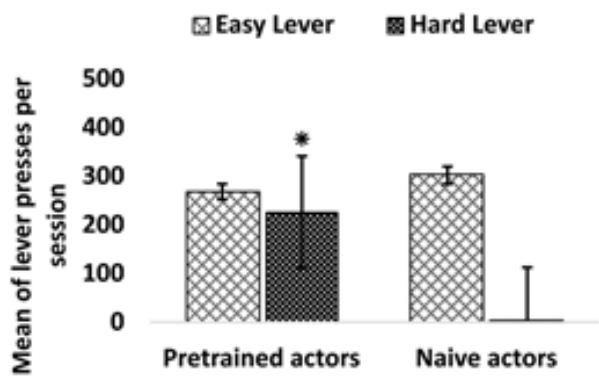

B) Vicarious Learning Phase

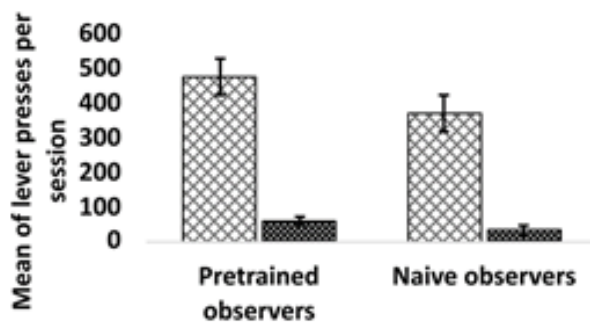

Note. A) Pretrained actors pressed more on the hard lever, thus choosing to share a reward with their congener more often compared to naive rats. B) Exposure to the prosocial phase had no impact on the behaviors of the observer rats once becoming actors. *denotes significance different from naive actors, $p<.05$. 
The descriptive statistics of lever presses indicated that, for the prosocial phase, pretrained actors pressed more on the hard lever $\left(M=227.06, \sigma_{\bar{x}}=17.95,95 \%\right.$ CI $\left.[191.71,262.41]\right)$ than naive actors $(M=$ $\left..53, \sigma_{\overline{\mathrm{x}}}=14.66,95 \% \mathrm{CI}[-28.33,29.39]\right)$, which pressed more on the easy lever $\left(M=303.38, \sigma_{\overline{\mathrm{x}}}=37.42\right.$, $95 \%$ CI $[229.71,377.05])$ than pretrained actors $\left(M=269.93 \sigma_{\bar{x}}=45.83,95 \%\right.$ CI $\left.[179.71,360.16]\right)$. Testing vicarious learning, observers from both pretrained and naive groups showed no differences in lever presses, both groups making significantly more easy $\left(M=475.92, \sigma_{\bar{x}}=50.85,95 \%\right.$ CI [375.82, 579.02]; $M=371.02$, $\sigma_{\overline{\mathrm{x}}}=41.79,95 \%$ CI $[288.76,453.28]$, respectively) than hard $\left(M=58.98, \sigma_{\overline{\mathrm{x}}}=19.92,95 \%\right.$ CI $[19.76,98.19]$; $M=31.92, \sigma_{\bar{x}}=16.37,95 \%$ CI [-0.30, 64.14], respectively) lever presses. Results showed that groups differed in terms of hard lever $\left(F(3,285)=34.882, p=.013\right.$; partial $\left.\eta^{2}=.269\right)$ and easy lever $(F(3,285)=$ $3.645, p<.001$; partial $\left.\eta^{2}=.037\right)$ presses. Games-Howell post-hoc tests showed that in the prosocial phase, the pretrained actors made significantly more hard lever presses compared to the naive actors $(p<.001$, $95 \%$ CI $[148.87,304.18])$. No differences were observed between the pretrained and the naive actors in easy lever presses ( $p=.911,95 \%$ CI [-164.91, 98.01]; see Figure 3A). For the vicarious learning phase, Games-Howell post-hoc tests showed no significant differences on the hard lever presses $(p=.761,95 \%$ CI $[-45.54,99.66])$ and the easy lever presses $(p=.473,95 \%$ CI $[-83.95,293.75])$ between pretrained and naive observers (see Figure 3B).

\section{Behavioral Analysis}

\section{Behavior Frequencies}

\section{Figure 4}

Pretraining Promotes Interaction and Communication Behaviors in Adolescent Rat Dyads
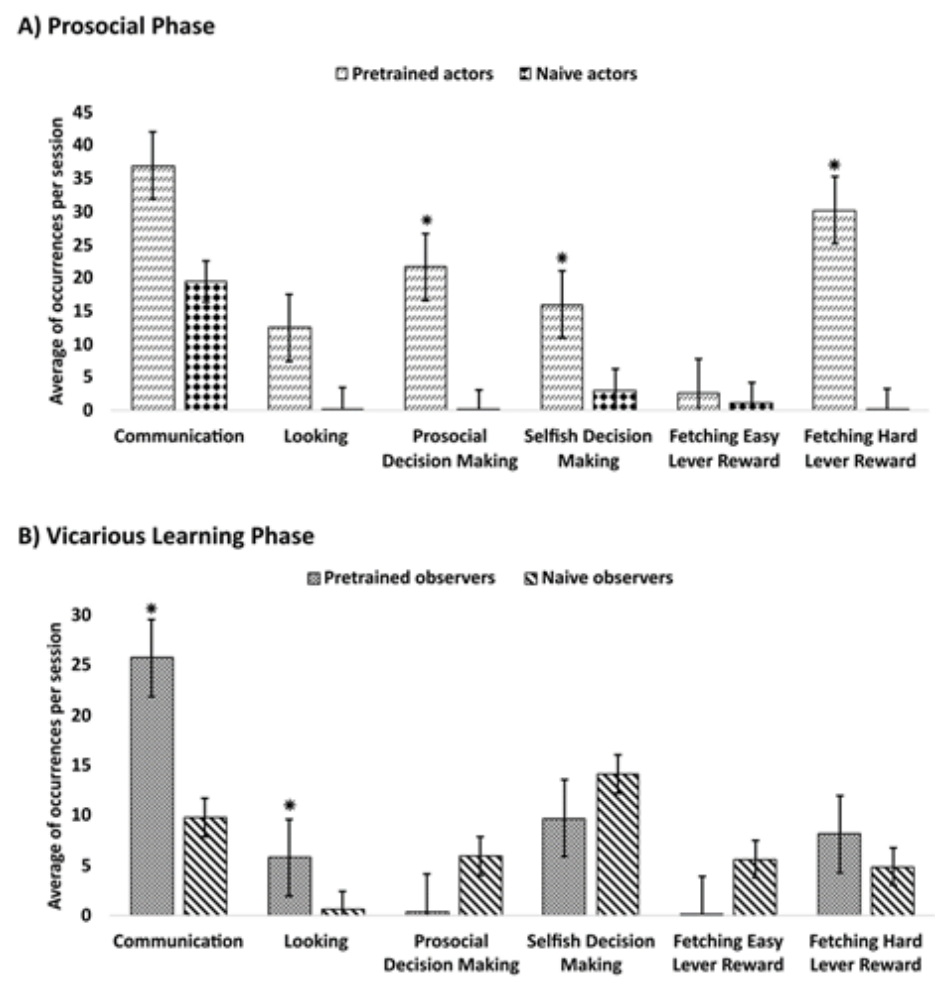

Note. A) During the prosocial sessions, pretrained actors made significantly more prosocial decision making than naive actors. Notably, observer rats in pretrained dyads reached their feeders significantly more often following hard lever presses by the actor, suggesting an understanding of the lever contingencies. B) Exposure to prosocial behavior did not influence prosocial behavior of observer rats. However, rats from pretrained dyads communicated and looked at each other more often during vicarious testing sessions than rats from naive dyads. *denotes significance, $p<.05$. 
A one-way MANOVA was used to determine the effect of a pretraining phase on behavior frequencies, treating the number of observations as units. See Figure 4A and B for visual representation of the data. Six behaviors were assessed (Communication, Looking, Prosocial decision making, Selfish decision making, Fetching hard lever reward, Fetching easy lever reward; see Table 1 for operational definition of these behavioral responses). For detailed descriptive statistics of all coded behavior, see Table 2 .

Table 2

Descriptive Statistics of All Coded Behaviors

\begin{tabular}{|c|c|c|c|c|c|c|}
\hline Behavior & Group & Frequency & Mean & Std. Error & $\begin{array}{c}95 \% \text { CI Lower } \\
\text { Bound }\end{array}$ & $\begin{array}{l}95 \% \text { CI Upper } \\
\text { Bound }\end{array}$ \\
\hline \multirow{4}{*}{$\begin{array}{l}\text { Communication - } \\
\text { Total duration }\end{array}$} & Pretrained actors & NA & 213.31 & 18.69 & 176.40 & 250.22 \\
\hline & Naive actors & NA & 258.07 & 21.11 & 216.37 & 299.78 \\
\hline & Pretrained observers & NA & 190.59 & 31.59 & 128.20 & 252.97 \\
\hline & Naive observers & NA & 80.20 & 23.48 & 33.82 & 126.57 \\
\hline \multirow{4}{*}{$\begin{array}{l}\text { Communication - } \\
\text { Total of Occurrences }\end{array}$} & Pretrained actors & 2218 & 36.96 & 1.70 & 33.60 & 40.32 \\
\hline & Naive actors & 915 & 19.46 & 1.92 & 15.67 & 23.26 \\
\hline & Pretrained observers & 542 & 25.81 & 2.87 & 20.13 & 31.48 \\
\hline & Naive observers & 372 & 9.78 & 2.13 & 5.56 & 14.01 \\
\hline \multirow{4}{*}{$\begin{array}{l}\text { Looking - } \\
\text { Total duration }\end{array}$} & Pretrained actors & NA & 12.21 & 1.47 & 9.30 & 15.12 \\
\hline & Naive actors & NA & 0.80 & 1.66 & -2.48 & 4.09 \\
\hline & Pretrained observers & NA & 14.84 & 2.49 & 9.92 & 19.76 \\
\hline & Naive observers & NA & 0.87 & 1.85 & -2.78 & 4.52 \\
\hline \multirow{4}{*}{$\begin{array}{l}\text { Looking - Total of } \\
\text { occurrences }\end{array}$} & Pretrained actors & 427 & 7.20 & 0.82 & 5.57 & 8.82 \\
\hline & Naive actors & 15 & 0.31 & 0.93 & -1.52 & 2.15 \\
\hline & Pretrained observers & 122 & 5.81 & 1.39 & 3.05 & 8.56 \\
\hline & Naive observers & 22 & 0.57 & 1.03 & -1.46 & 2.62 \\
\hline \multirow{4}{*}{$\begin{array}{l}\text { Prosocial Decision } \\
\text { Making }\end{array}$} & Pretrained actors & 1303 & 21.71 & 2.93 & 15.92 & 27.51 \\
\hline & Naive actors & 3 & 0.06 & 3.31 & -6.48 & 6.61 \\
\hline & Pretrained observers & 7 & 0.33 & 4.96 & -9.46 & 10.13 \\
\hline & Naive observers & 226 & 5.94 & 3.68 & -1.33 & 13.23 \\
\hline \multirow{4}{*}{$\begin{array}{l}\text { Selfish Decision } \\
\text { Making }\end{array}$} & Pretrained actors & 959 & 15.98 & 2.29 & 11.45 & 20.50 \\
\hline & Naive actors & 147 & 3.12 & 2.59 & -1.98 & 8.24 \\
\hline & Pretrained observers & 204 & 9.71 & 3.87 & 2.06 & 17.36 \\
\hline & Naive observers & 537 & 14.13 & 2.88 & 8.44 & 19.81 \\
\hline \multirow{4}{*}{$\begin{array}{l}\text { Fetching Hard Lever } \\
\text { Reward }\end{array}$} & Pretrained actors & 1812 & 30.20 & 2.33 & 25.58 & 34.81 \\
\hline & Naive actors & 12 & 0.25 & 2.64 & -4.96 & 5.47 \\
\hline & Pretrained observers & 171 & 8.14 & 3.95 & 0.33 & 15.94 \\
\hline & Naive observers & 38 & 4.89 & 2.93 & -0.90 & 10.69 \\
\hline \multirow{4}{*}{$\begin{array}{l}\text { Fetching Easy Lever } \\
\text { Reward }\end{array}$} & Pretrained actors & 161 & 2.68 & 0.98 & 0.74 & 4.62 \\
\hline & Naive actors & 53 & 1.12 & 1.10 & -1.06 & 3.31 \\
\hline & Pretrained observers & 2 & 0.09 & 1.65 & -3.18 & 3.37 \\
\hline & Naive observers & 214 & 5.63 & 1.23 & 3.19 & 8.06 \\
\hline
\end{tabular}

Preliminary assumption checking revealed that data were normally distributed, as assessed by a Normal Q-Q plot. There were no univariate outliers in the data, as assessed by inspection of a boxplot for values greater than 1.5 box-lengths from the edge of the box and 11 multivariate outliers assessed by the Mahalanobis distance $(p<.001)$. After analysis with and without these 11 outliers, they were kept in the 
data as they did not affect the results of the one-way MANOVA. There were linear relationships, as assessed by scatterplot and no multicollinearity $(r<.90, p<.001)$. There was no homogeneity of variance-covariance matrices, as assessed by Box's M test $(p<.001)$. Because this assumption was not fulfilled, a Pillai's Trace effect was used instead of a Wilks' $\Lambda$. Additionally, since the Levene's test of equality of variances was significant $(p<.05)$, a lower alpha level of $.025(p<.025)$ was used.

Analysis revealed significant difference in behavioral responses between groups $(p<.020)$. GamesHowell post-hoc tests showed that there was a significant difference between pretrained and naive dyads $(p$ $<.024)$ for all analyzed behaviors. More precisely, for the prosocial phase, pretrained dyads communicated more often $(p<.001)$, looked at each other more often and for longer periods of time $(p<.001)$ and had more prosocial decision making $(p<.021)$ than naive dyads (see Figure 4A). As for the vicarious learning phase, the naive dyads communicated less and for shorter periods of time $(p<.003)$ and looked less often and for shorter periods of time at each other compared to pretrained dyads ( $p<.001$; see Figure 4B).

\section{Transitional Probability of Behavioral Responses}

Transitional probability analyses were performed using O'Connor's SEQGROUPS SPSS syntax program (O'Connor, 1999) between naive and pretrained dyads. Data logging of six behaviors during the 16 sessions of the prosocial phase (i.e., Communication, Looking, Prosocial decision making, Selfish decision making, Fetching hard lever reward, Fetching easy lever reward). See Figure 5 A and B for a visual representation of the data. An assumption to perform the transitional probability analysis is a confirmation of behavioral interdependence in each of the groups. The analysis revealed that the likelihood ratio chi-square values reached significance for the pretrained actors $\left(\chi^{2}(25,7205)=[83.3], p\right.$ $<.001)$, naive actors $\left(\chi^{2}(25,1157)=[221.67], p<.001\right)$, pretrained observers $\left(\chi^{2}(25,572)=[403.86], p\right.$ $<.001)$ and naive observers $\left(\chi^{2}(25,1556)=[1160.41], p<.001\right)$, indicating an interdependence in the transition of behaviors within the naive and the pretrained groups. Incidentally, the likelihood ratio indicates the percentage of which a behavior might follow another (e.g., $\mathrm{X} \%$ that the behavior " $\mathrm{A}$ " follows behavior " $\mathrm{B}$ ") but is not indicative of the number of occurrences of the targeted behavior. For instance, the naive group displayed 915 communication behaviors over the 16 testing sessions, and in this group, there was an $88 \%$ probability that a communication behavior was followed by another communication. In contrast, the pretrained group communicated 2218 times over the 16 sessions but this group showed only a $37 \%$ probability for the same behavior (see Figure 5).

In this context, the transitional probability analysis examined sequences of behavioral responses from the actor to the observer rat, and vice versa. Analyses of behavioral responses of the pretrained dyads in the prosocial phase revealed a $37.44 \%(z=7.34 ; p<.001)$ probability that communication would be followed by a second communication behavior. Moreover, the probability for a prosocial decision being followed by a communication was $28.94 \%(z=-2.22 ; p=.02)$ and $28.18 \%$ for that behavior being followed by the observer fetching a reward in its feeder $(z=2.20 ; p=.02)$, suggesting that the observers associated a hard lever press by the actor with a reward pellet gain. Following fetching behavior, the probability for a communication between dyads was $28.26 \%(z=-3.51 ; p<.001$; see Figure 5).

As for the naive actors in the prosocial phase, the transitional probability of communication following any other behavior was very low (Looking: $z=-1.96 ; p=.04$, Prosocial Decision Making: $z=$ 2.03; $p=.04$, Selfish Decision Making: $z=-8.57 ; p<.001$, Fetching Easy Lever Reward: $z=-8.62 ; p<$ .001 , Fetching Hard Lever Reward: $z=-4.08 ; p<.001$ ) except for a communication being followed by another communication $(88.03 \% ; z=13.52 ; p<.001)$. Moreover, communication was rarely preceded by either the prosocial $(0.11 \% ; z=-2.03 ; p=.0420)$ or selfish decision making $(8.52 \% ; z=-8.578 ; p<.001)$. After a prosocial decision making, the probability for the observer to go towards its feeder was higher $(66.67 \% ; z=11.23 ; p<.001)$, suggesting that observers associated a hard lever press by the actor with a reward. The transitional probability also revealed many behavior omissions, such as the probability that a prosocial decision being followed by a communication was completely absent from the behavior sequences $(p<.001$; see Figure 5 for all behavior omissions). 


\section{Figure 5}

Transitional Probabilities in Behavioral Responses in Pretrained vs Naive Actors (Prosocial Phase)

A) Pretrained Actors

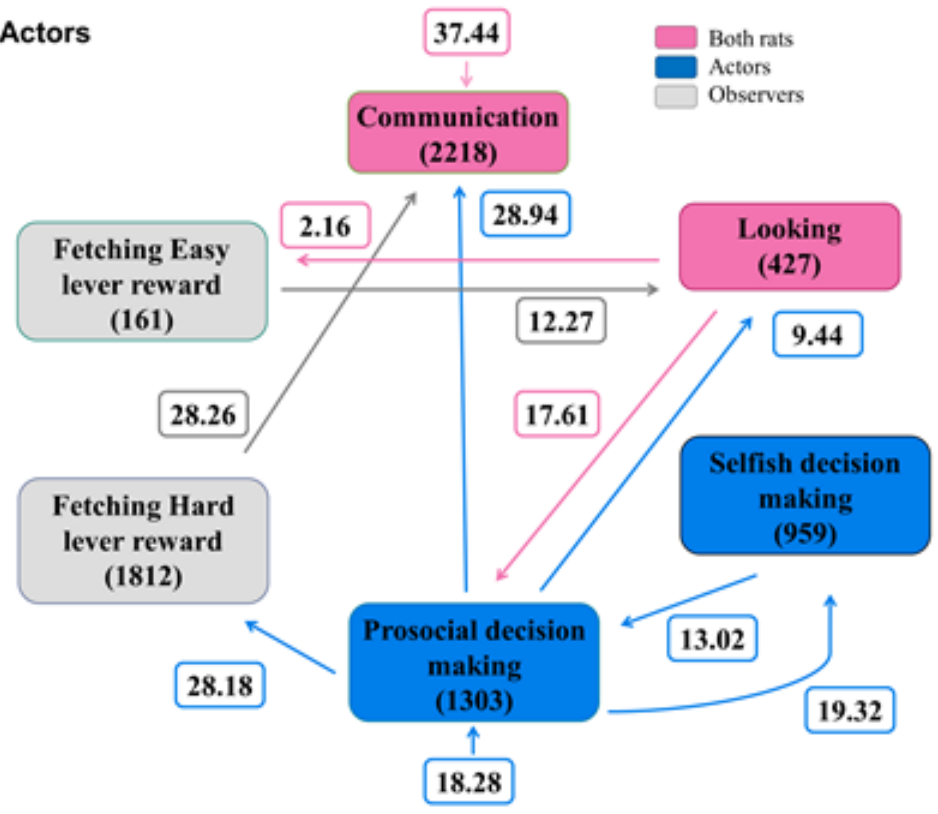

B) Naive Actors

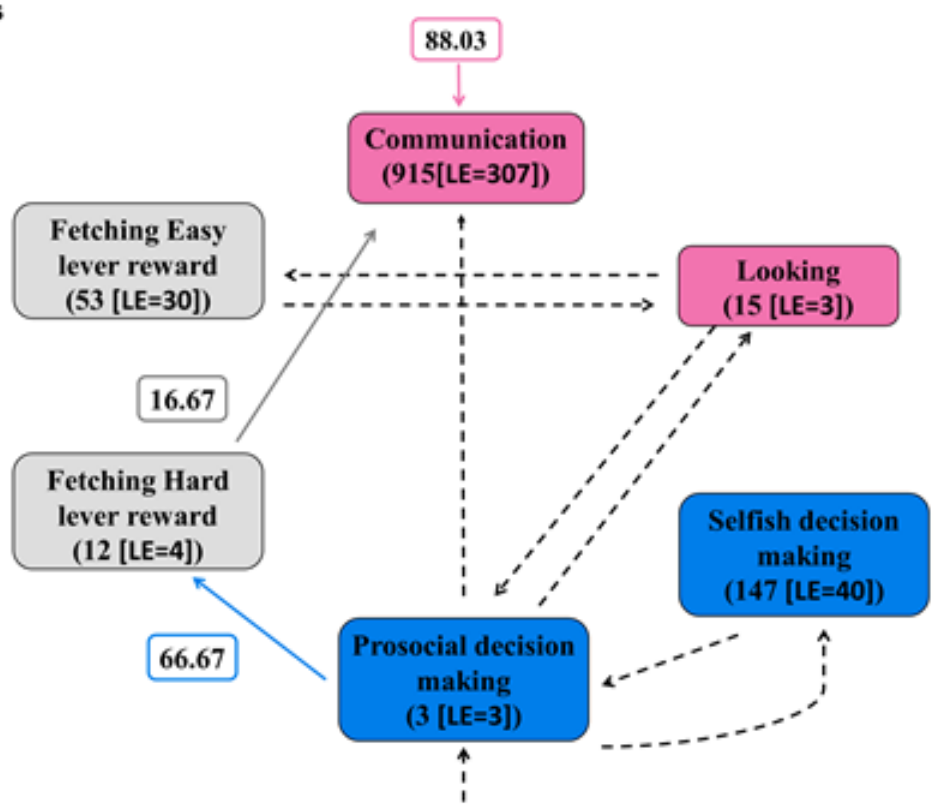

Note. Pretrained actors (A) showed behavioral diversity and complex behavioral sequences compared to naive actors (B). This is manifest through significant differences in frequencies of the logged behaviors (indicated in parentheses) and by arrows indicating probabilities (in \%) for one behavior to be related to another one (e.g., fetching hard lever being followed by communication, etc.) Dotted lines indicate the absence of behavioral relationships in the naive dyads. LE = Long-Evans.

As for the vicarious phase (see Figure 6A and B), there was a probability of $83.33 \%(z=4.84 ; p<$ $.001)$ that the pretrained observers would retrieve their reward after the actor made a prosocial decision by pressing the hard lever. This could suggest that the observers understood the relationship between a hard lever press and the delivered reward of one sucrose pellet (see Fig. 6A). For the group of naive observers, the probability for a communication to be followed by another communication was elevated $(74.19 \%, z=$ 
26.12; $p<.001$; see Figure 6B). However, there were very low probabilities for communication between naive dyads to be followed by any other monitored behaviors (Fetching easy lever reward: $7.26 \% ; z=-$ 4.17, $p<.001$; Fetching hard lever reward: $0.81 \% ; z=-7.59 ; p<.001$; Looking: $0.27 \%$; $z=-2.14, p=.03$; Prosocial decision making: $1.61 \% ; z=-8.10, p<.001$; Selfish decision making: $15.86 \% ; z=-8.67, p<$ $.001)$. Following a selfish decision making, the probability of pressing the easy lever again was elevated $(51.64 \% ; z=5.60 ; p<.001)$ compared to the probability of a prosocial decision making - i.e., pressing on the hard lever $(6.57 \% ; z=-3.50 ; p<.001)$. These findings indicate that the responses of the observers once becoming actors were not influenced by their association with a pretrained or naive actor. Our findings did not support vicarious learning.

\section{Figure 6}

Transitional Probabilities in Behavioral Responses in Pretrained vs Naive Observers (Vicarious Phase)

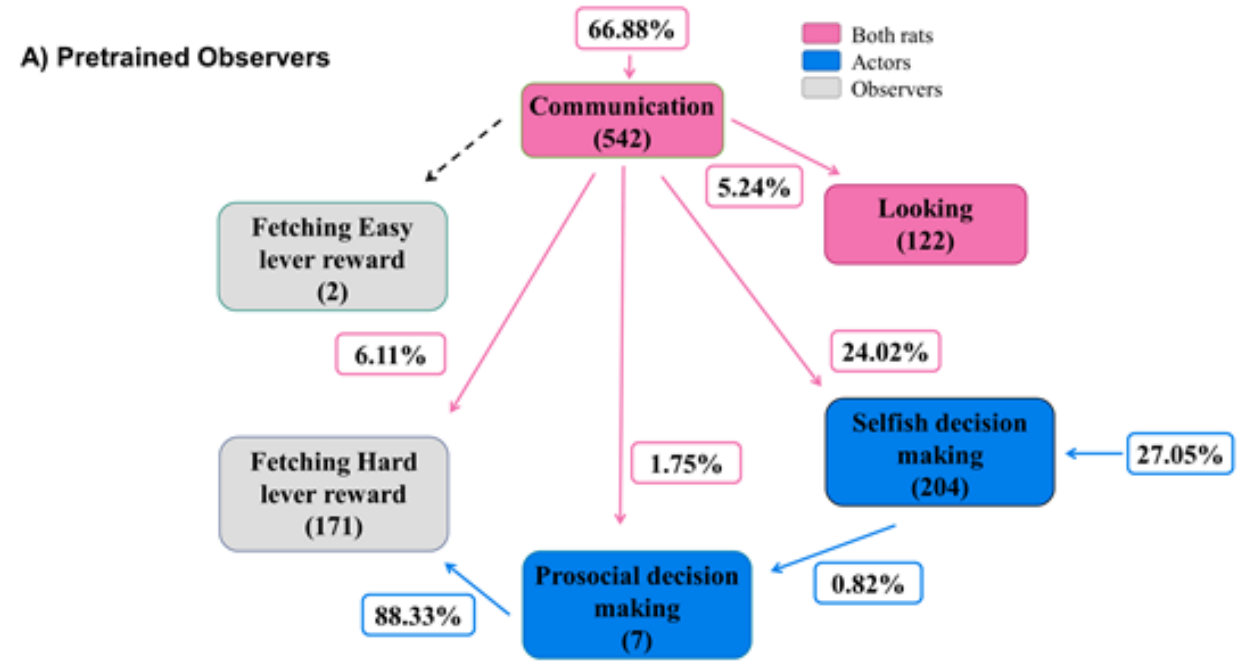

B) Naive Observers

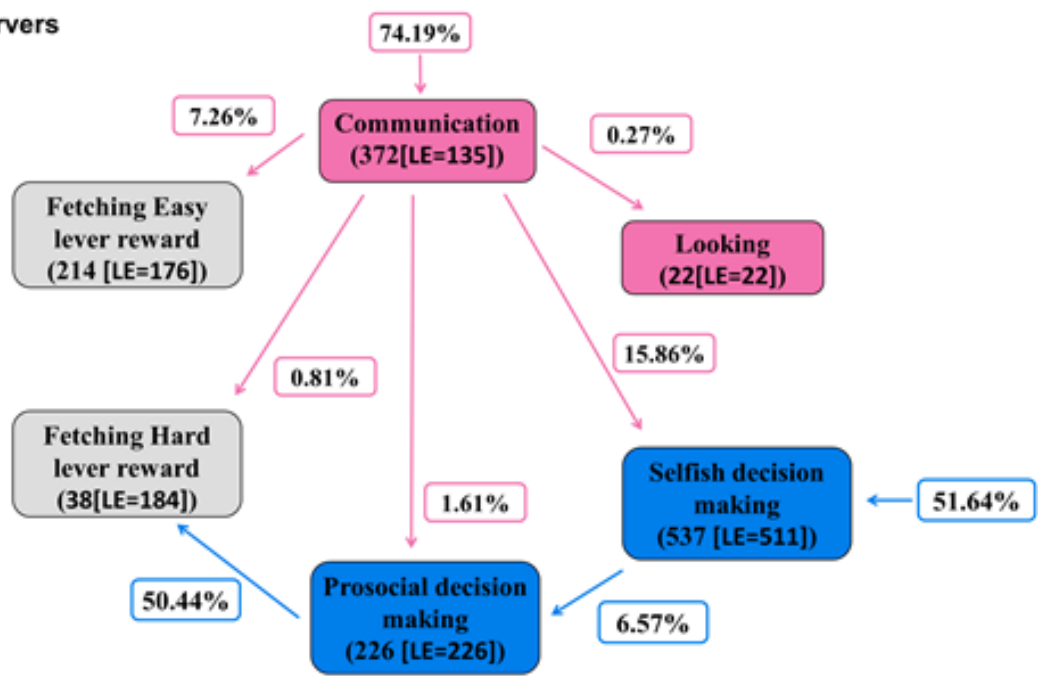

Note. Pretrained observers (A) and naive observers (B) both showed fewer behavioral diversity than rats in the prosocial phase (Figure 5A and B). (B) Long-Evans did the entirety of the prosocial decision making, and nearly all of the selfish decision making, showing more activity than Sprague-Dawley. Dotted lines indicate the absence of behavioral relationships in the dyads. LE $=$ LongEvans. 


\section{Strain Differences}

An independent-samples $t$-test was run to determine if there were differences between Long-Evans (LE) and Sprague-Dawley's (SD) performance in the naive groups. The data was normally distributed, as assessed by a Normal Q-Q plot and there were five outliers as assessed by inspection of a boxplot. After inspection, these 5 outliers were removed from the data due to unusually high lever presses. The assumption of homogeneity of variances was rejected, as assessed by Levene's test for equality of variances $(p<.001)$. Therefore, the Welch $\mathrm{t}$ Test Statistic was used. Sprague-Dawley rats pressed more on the easy lever than Long-Evans rats did $(M=383.93, \sigma \overline{\mathrm{x}}=438.89,95 \%$ CI [303.71, 464.14]; $M=233.46, \sigma \overline{\mathrm{x}}=225.08,95 \%$ CI [175.53, 291.38], respectively). As for the hard lever, no differences were found between Long-Evans and Sprague-Dawley rats $(M=6.64, \sigma \overline{\mathrm{x}}=20.75,95 \%$ CI $[1.30,11.98] ; M=4.40, \sigma \overline{\mathrm{x}}=15.80,95 \%$ CI [1.51, 7.28], respectively). A significant difference was found in easy lever presses between the LE and the SD, $t(167)=-2.89, p<.001,95 \%$ CI $[29.29,271.64]$ but not in hard lever presses, $t(167)=.703, p=.37,95 \%$ CI [-3.87, 8.35].

An independent-samples $t$-test further examined differences between naive LE and SD's behavioral responses. The data was normally distributed, as assessed by a Normal Q-Q plot and there was no outlier as assessed by inspection of a boxplot. The assumption of homogeneity of variances was rejected, as assessed by Levene's test for equality of variances $(p<.001)$. Therefore, the Welch $\mathrm{t}$ Test Statistic was used. Analyses showed no differences between the strains for the mean occurrences of communication behaviors $t(57.35)=.469, p=.641,95 \%$ CI $[-3.14,5.06]$, or mean communication duration $t(83)=.005, p$ $=.996,95 \%$ CI $[-72.59,72.97]$. There were also no differences in occurrences of looking, $t(29.97)=1.98$, $p=.056,95 \%$ CI $[-0.01,1.38]$, but a difference was found in looking duration, $t(35.41)=2.08, p=.045$, $95 \%$ CI $[0.02,2.07]$, LE looking at each other for longer time periods than SD rats. Furthermore, LE engaged in more prosocial and selfish decision making compared to SD rats, $t(27.04)=2.11, p=.043,95 \%$ CI $[0.25,16.10] ; t(27.81)=3.30, p=.003,95 \%$ CI [6.60, 28.09], respectively. As for observer rats, LE approached feeders more frequently when the actor pressed the hard and the easy levers than SD rats, $t(27.04)=2.29, p=.030,95 \%$ CI $[0.70,12.37] ; t(28.25)=2.75, p=.010,95 \%$ CI $[1.60,10.96]$, respectively.

\section{Long-Evans’'Activity Does Not Impact the Naïve Group}

An independent-samples $t$-test further examined differences between Pretrained and Naive LE's behavioral responses. The data were normally distributed, as assessed by a Normal Q-Q plot and there was no outlier as assessed by inspection of a boxplot. The assumption of homogeneity of variances was rejected, as assessed by Levene's test for equality of variances $(p<.001)$. Therefore, the Welch $\mathrm{t}$ Test Statistic was used. Analyses comparing pretrained and naive LE rats revealed that exposure to pretraining sessions led to increased behavioral responses (communication $t(74)=5.33, p<.001,95 \%$ CI $[11.08,24.47]$; Looking: $t(74)=5.34, p<.001,95 \%$ CI $[4.38,9.63]$; Prosocial decision making: $t(74)=4.77, p<.001,95 \%$ CI $[12.50,30.55]$; Selfish decision making: $t(74)=5.03, p<.001,95 \%$ CI $[8.13,18.82]$; Fetching hard lever reward: $t(74)=8.83, p<.001,95 \% \mathrm{CI}[23.16,36.73])$. Only fetching the easy lever reward was not affected $t(74)=0.35, p=.727,95 \% \mathrm{CI}[-3.79,5.41]$. Consistently, analyses showed pretrained LE rats to press more on both levers compared to naive LE rats (Easy: $M=269.93, \sigma_{\bar{x}}=37.43,95 \%$ CI [260.45,279.40], $M$ $=162.31, \sigma_{\overline{\mathrm{x}}}=27.04,95 \%$ CI [149.06, 175.55], $[t(94)=2.33, p=.022]$; Hard: $M=227.06, \sigma_{\overline{\mathrm{x}}}=29.42,95 \%$ CI $[219,61,234,50], M=0.65, \sigma_{\bar{x}}=0.36,95 \%$ CI $\left.[0.47,0.82],[t(94)=7.69, p<.001]\right)$.

\section{Discussion}

Using a non-aversive protocol, this study demonstrates the contribution of pretraining to increase occurrences of behaviors associated to prosociality in an operant paradigm and its impact to regulate behavioral interactions between adolescent rat dyads. This study is the first to show that pretraining is associated with increased interactions between actor and observer rats, which influence goal directed behaviors in the actor rats. Our findings failed to support vicarious learning in observer rats of the pretrained 
or naive groups, suggesting that observation even in a rewarding context was not sufficient to foster behavioral emulation of prosocial-like behavior. Consistent with previous studies (Andrews et al., 1995; Gökçek-Saraç et al., 2015; Stryjek et al., 2013), Long-Evans rats show increased activity and display more prosocial behaviors compared to Sprague-Dawley rats. This study helps to clarify the role of behaviors in prosociality, more specifically the role of communication patterns in a dyad based paradigm. Considering that the adolescent period is characterized by many cerebral and behavioral changes as well as intense social play, it is an interesting period to assess prosociality in rat dyads (Dahl et al., 2018). Marked by significant brain plasticity, adolescent exposure to different environmental settings (enrichment or stress) has been shown to influence adult responses (both behavioral and biochemical responses; Cotella et al., 2019). This paradigm likely benefits from sensitivity observed at the adolescence period, which is likely to promote further understanding of brain processes contributing to prosocial behavior. This study demonstrates that adolescent exposure to pretraining can impact behavioral responses in late adolescence/early adulthood, likely impacting neurochemical responses. These findings pave the way for future research on prosocial behaviors in adolescent rats and possible consequences on cerebral development and adulthood.

\section{Pretraining Strongly Influences Actor's Prosocial-like Behavioral Expression}

Our findings indicate that lever discrimination acquired during the pretraining phase is an essential element in developing delayed prosocial-like responses towards a congener. This is evident through elevated hard lever presses in the pretrained compared to the naive groups, a response associated with significantly increased rewards provided to observer rats in pretrained compared to non-trained dyads. While the naive group was composed of two strains (see strain differences section below), when pretrained Long-Evans were compared to naive Long-Evans only (i.e., excluding Sprague-Dawley rats from analyses), results showed that pretrained Long-Evans pressed more on both levers and engaged in more behaviors than their naive congeners. These findings further support the pretraining to act as an essential element in prosocial-like responses. Looking at the behavioral sequences, our findings support different behavioral interactions in naive and pretrained dyads. Results from transitional probabilities revealed that pretrained rats displayed a more complex set of behaviors compared to the naive dyads, marked by increased communication that could influence fetching behavior and associated with increased probability of recurrent prosocial hard lever press (see Figure 5A and B). Indeed, the pretrained actors' decision-making process was marked by more frequent prosocial decision making (i.e., an initial approach towards an easy lever ending in a hard lever press). Moreover, the transitional probability analysis also supports prosocial decision making to initiate periods of communication that promoted more interactions (e.g., communication, looking) in the pretrained actors (see Figure 5A). This may suggest that a form of feedback may have happened after the prosocial decision making. Rats have the ability to communicate their positive affect such as a prosocial affiliative call and appetitive state at a frequency of 50 kilohertz (Engelhardt et al., 2018; Seffer et al., 2014). Indeed, Łopuch and Popik (2011) found that rats tend to communicate ultrasonically in a paired operant task using the 50 kilohertz frequency. Based on these findings, it is probable that the observed interaction involved a form of ultrasonic communication destined to convey their positive or cooperative feelings to one another. Finally, the observed behaviors in the pretrained actors appears related to pretraining, and the fact that these actors remained in a familiar environment where they had been exposed to the levers' function and differential reward attributes (Horner et al., 2013).

Interestingly, naive actors had lower behavior frequencies and more rigid behavior sequences compared to pretrained rats (see Figure 5B). Communication followed by any other behaviors was also lower according to the transitional probability analysis. Furthermore, naive actors had three missing relationships between behaviors, all surrounding communication, that were present in the pretrained group (see Figure 5A and B). These missing relationships can inform us that the naive actors had fewer behaviors and significantly lower communication, which according to results presented above, could be related to the lack of a pretraining phase and thus help explain the discrepancy between the pretrained and naive rats task performances. 
Our observations strongly support task contingencies learned during the pretraining phase (e.g., familiarization of the rats to the operant box and the lever press-associated rewards) to play a critical role in the observation of learned prosocial-like behaviors using an operant task. Specifically, the 14-day pretraining session (using a double reward upon a hard lever press as recommended by Brady and Floresco, 2015) helped discriminate the two levers and acted to minimize generalization between the pretraining and the prosocial phase (Horner et al., 2013; Oomen et al., 2013). Other studies assessing empathy in rats using aversive conditions also support the importance of repeated exposure to a setting and control gained over a situation as a key factor influencing prosocial behavior- an 'actor' rat is indeed shown to need a series of exposures to a trapped congener for it to gain the ability to rescue the congener rat (Ben-Ami Bartal et al., 2011). Thus, prosociality requires familiarity to express itself. Our findings further demonstrate that prosocial responses are accompanied by singular behavioral responses between the rat dyads. These findings support the contextual setting to significantly influence an animal's disposition to attend to a congener.

\section{Pretraining had no Impact on Vicarious Learning}

Our findings failed to confirm that rats observing a pretrained congener performing a task would subsequently learn to display prosocial behaviors by vicarious observation. Interestingly, rats from the vicarious learning phase demonstrated an understanding of the task contingencies as shown by the ability to press on both levers (see Figure 3B) and by appropriately timed reward retrieval (fetching hard lever reward) (see Figure 4A). While rats seemed to understand task conditions through vicarious learning, as seen in previous literature (Ben-Ami Bartal et al., 2011; Hernandez-Lallement et al., 2015; Meyza et al., 2017; Yamada \& Sakurai, 2018), a novel finding of this study is the lack of prosocial behaviors displayed by the pretrained and naive observers (see Figure 6). Our results support the ability for rats to display prosocial-like behaviors, but only in specific scenarios where rats are directly exposed to task contingencies such as lever pressing, door opening or entering a specific compartment (Ben-Ami Bartal et al., 2011; Hernandez-Lallement et al., 2015). In contrast, although observer rats showed task contingency understanding through fetching behaviors and lever presses, they failed to display the same level of overall behaviors compared to the actors that received the pretraining. These results could indicate that vicarious learning of a prosocial task is not enough to trigger a prosocial-like response in rats and that a pretraining is a necessity for it to be triggered.

Whereas our findings indicate the inability for rats to learn prosocial-like behaviors by vicarious observation, behavior frequencies revealed that observers that learned from pretrained rats communicated and looked at each other more often and for longer periods of time compared to naive observers (see Figure 4B). Furthermore, naive observers' results revealed that the probability of any behavior following a communication was reduced compared to pretrained observers, and communication frequencies and duration were significantly lower than pretrained observers (see Figure 6). These results may be related to learning from naive actors (i.e., that did not have a pretraining phase), having been exposed to reduced learning opportunities, which hindered their performance.

Interestingly, behavioral analysis of the observer during the prosocial phase showed that it was able to anticipate reward. When the pretrained actor pressed on the hard lever, the probability for the observer to fetch its reward in the feeder was $28 \%$ versus $1.70 \%$ following an easy lever press, indicating that the pretrained observers learned to discriminate the levers' dispensed rewards (see Figure 5A). A similar response was noted in the naive dyads, although the small number of hard lever presses in this condition render interpretation more hazardous. Therefore, although animals showed some understanding of the task contingencies, witnessing a pretrained or naive actor did not transfer to prosocial responses. This may indicate that the observation of a pretrained actor is not sufficient to learn a task, and that being actively exposed to a pretraining phase is necessary to generate prosocial-like responses. 


\section{Long-Evans Display More Diverse Set of Behaviors}

Long-Evans and Sprague-Dawley rats have commonly been used in social/behavioral paradigms (Ben-Ami Bartal et al., 2011; Hernandez-Lallement et al., 2015). In this study, independent t-tests supported strain differences in the number of easy presses in the naive group. Our findings indicate that SpragueDawley rats pressed more on the easy lever than Long-Evans. This appears related to Long-Evans rats showing an overall increased general activity than Sprague-Dawley, translating in reduced time spent pressing the easy lever and increased time interacting with the congener and exploring the hard lever. Indeed, analyses of behavior frequencies support the idea that Long-Evans rats engage in more behaviors than Sprague-Dawley, spending longer periods looking at each other and more decision making towards both levers. Additionally, Long-Evans were generally more active, displaying higher frequencies of behaviors than Sprague-Dawley rats (e.g., engaging in more prosocial decision making, observers going more often to their feeders). These results contrast Ku and colleagues' (2016) findings supporting juvenile Sprague-Dawley rats to engage more frequently with a congener and for longer periods of time compared to Long-Evans rats. This difference could partly be explained by the fact that pigmented (Long-Evans) rats tend to be more active during daytime than albino rats (Sprague-Dawley, Stryjek et al., 2013). Therefore, our results support research on Long-Evans' aptitude and their suitability for operant paradigms (Andrews et al., 1995), and confirm our hypothesis of enhanced performance of Long-Evans compared to SpragueDawley using the proposed model.

\section{Limitations and Future Directions}

The data although preliminary, support the learning of prosocial-like responses to be accompanied by singular communication patterns likely regulated through discrete biochemical changes. Similar to Hernandez-Lallement et al. (2015), our study has defined prosociality through behavioural responses expressed in a social environment in which one party acts in a helping manner towards a congener. Using a paradigm assessing the presence of prosocial-like behaviours in an environmental context where choices were dichotomous (prosocial versus selfish; prosocial responses involved an extra effort), our findings support pretraining as essential to prosocial-like responses, and vicariant learning sessions as insufficient to promote prosocial choices. As such, one hypothesis brought forward by this study is the role of a pretraining phase as one possible explanation on the expression of prosocial-like behaviours and not necessarily the presence of prosociality itself. It is important to note that a limiting factor and unknown mediator of performance surrounding the role of the pretraining phase is the possible extinction phase that may have occurred with the hard lever due to phase-dependant differentiated rewards for the hard lever. Additional work is required to further characterize biobehavioral relationship regulating prosocial-like behaviors and vicarious learning in rodents. Thus, a study including males and females is warranted to further validate this model. Subsequent studies would also benefit from including recording of the rats' ultrasonic vocalization, a limiting factor in our analyses. This would help interpret the nature of the interaction between the actors and observers. Many studies have explored ultrasonic communication in rats and have found it to be a reliable metric to assess socio-emotional states (Seffer et al., 2014). Furthermore, future studies should consider a study in which all groups would be exposed to a pretraining phase, best controlling for familiarity with the context and task contingencies. This will allow a more in-depth analysis of behaviors and performance. Lastly, we found levers to act as a limiting physical factor as the pressure needed for the hard lever was set at 75 grams. Testing rats during the adolescence and early adulthood periods, the pressure needed to trigger a response is important considering their weight. It can thus be hard for the younger rat to maintain regular presses. As the rat gains weight, the pressure to body weight ratio diminishes and may no longer influence press rate. As explored by Oomen and colleagues (2013), a touch screen could be an alternative to the physical levers and thus is worth exploring in future studies.

Conflict of Interest: The authors declare no conflicts of interest. 


\section{Acknowledgements}

The authors would like to thank Tama Davis, Jacky Liang and Emerson Donnelly Ferguson for the animal care, technical support and guidance. The authors declare no conflict of interests. VC is supported by scholarships from the Québec Research Funds in Nature and Technologies. This work was supported by a discovery grant from the National Science and Engineering Research Council (NSERC) of Canada (RG203596-13) to HP.

\section{References}

Andrews, J. S., Jansen, J. H. M., Linders, S., Princen, A., \& Broekkamp, C. L. E. (1995). Performance of four different rat strains in the autoshaping, two-object discrimination, and swim maze tests of learning and memory. Physiology \& Behavior, 57(4), 785-790. https://doi.org/10.1016/0031-9384(94)00336-X

Achterberg, E. J. M., van Swieten, M. M. H., Houwing, D. J., Trezza, V., \& Vanderschuren, L. J. M. J. (2019). Opioid modulation of social play reward in juvenile rats. Neuropharmacology, 159, 107332. https://doi.org/10.1016/j.neuropharm.2018.09.007

Atsak, P., Orre, M., Bakker, P., Cerliani, L., Roozendaal, B., Gazzola, V., Moita, M., \& Keysers, C. (2011). Experience modulates vicarious freezing in rats: A model for empathy. PLoS ONE, 6(7). https://doi.org/10.1371/journal.pone.0021855

Bem, T., Jura, B., Bontempi, B., \& Meyrand, P. (2018). Observational learning of a spatial discrimination task by rats: Learning from the mistakes of others? Animal Behaviour, 135, 85-96. https://doi.org/10.1016/j.anbehav.2017.10.018

Ben-Ami Bartal, I. B-A., Decety, J., \& Mason, P. (2011). Helping a cagemate in need: Empathy and pro-social behavior in rats. Science, 334(6061), 1427-1430. https://doi.org/10.1126/science.1210789

Ben-Ami Bartal, I. B_A., Rodgers, D. A., Bernardez Sarria, M. S., Decety, J., \& Mason, P. (2014). Pro-social behavior in rats is modulated by social experience. ELife, 3, 1-16. https://doi.org/10.7554/eLife.01385

Brady, A. M., \& Floresco, S. B. (2015). Operant procedures for assessing behavioral flexibility in rats. Journal of Visualized Experiments: JoVE, 96, 1-13. https://doi.org/10.3791/52387

Burke, A. R., McCormick, C. M., Pellis, S. M., \& Lukkes, J. L. (2017). Impact of adolescent social experiences on behavior and neural circuits implicated in mental illnesses. Neuroscience \& Biobehavioral Reviews, 76, 280 300. https://doi.org/10.1016/j.neubiorev.2017.01.018

Caballero, A., Granberg, R., \& Tseng, K. Y. (2016). Mechanisms contributing to prefrontal cortex maturation during adolescence. Neuroscience \& Biobehavioral Reviews, $40, \quad 4-12$. https://doi.org/10.1016/j.neubiorev.2016.05.013

Carlier, P., \& Jamon, M. (2006). Observational learning in C57BL/6j mice. Behavioural Brain Research, 174(1), 125131. https://doi.org/10.1016/j.bbr.2006.07.014

Church, R. M. (1959). Emotional reactions of rats to the pain of others. Journal of Comparative and Physiological Psychology, 52(2), 132-134. https://doi.org/10.1037/h0043531

Cronin, K. A. (2012). Prosocial behaviour in animals: The influence of social relationships, communication and rewards. Animal Behaviour, 84(5), 1085-1093. https://doi.org/10.1016/j.anbehav.2012.08.009

Cotella, E. M., Gomez, A. S., Lemen, P., Chen, C., Fernández, G., Hansen, C., Herman, J. P., \& Paglini, M. G. (2019). Long-term impact of chronic variable stress in adolescence versus adulthood. Progress in NeuroPsychopharmacology \& Biological Psychiatry, 88, 303-310. https://doi.org/10.1016/j.pnpbp.2018.08.003

Dahl, R. E., Allen, N. B., Wilbrecht, L., \& Suleiman, A. B. (2018). Importance of investing in adolescence from a developmental science perspective. Nature, 554(7693), 441-450. https://doi.org/10.1038/nature25770

Engelhardt, K.-A., Schwarting, R. K. W., \& Wöhr, M. (2018). Mapping trait-like socio-affective phenotypes in rats through $50-\mathrm{kHz}$ ultrasonic vocalizations. Psychopharmacology, 235(1), 83-98. https://doi.org/10.1007/s00213-017-4746-y

Friard, O., \& Gamba, M. (2016). BORIS: A free, versatile open-source event-logging software for video/audio coding and live observations. Methods in Ecology and Evolution, 7(11), 1325-1330. https://doi.org/10.1111/2041210X.12584

Greene, J. T. (1969). Altruistic behavior in the albino rat. Psychonomic Science, 14(1), 47-48. https://doi.org/10.3758/BF03336420

Gökçek-Saraç, Ç., Wesierska, M., \& Jakubowska-Doğru, E. (2015). Comparison of spatial learning in the partially baited radial-arm maze task between commonly used rat strains: Wistar, Sprague-Dawley, Long-Evans, and 
outcrossed Wistar/Sprague-Dawley. Learning \& Behavior, 43(1), 83-94. https://doi.org/10.3758/s13420014-0163-9

Hernandez-Lallement, J., van Wingerden, M., Marx, C., Srejic, M., \& Kalenscher, T. (2015). Rats prefer mutual rewards in a prosocial choice task. Frontiers in Neuroscience, 8, 1-9. https://doi.org/10.3389/fnins.2014.00443

Horner, A. E., Heath, C. J., Hvoslef-Eide, M., Kent, B. A., Kim, C. H., Nilsson, S. R. O., Alsiö, J., Oomen, C. A., Holmes, A., Saksida, L. M., \& Bussey, T. J. (2013). The touchscreen operant platform for testing learning and memory in rats and mice. Nature Protocols, 8(10), 1961-1984. https://doi.org/10.1038/nprot.2013.122

Ku, K. M., Weir, R. K., Silverman, J. L., Berman, R. F., \& Bauman, M. D. (2016). Behavioral phenotyping of juvenile Long-Evans and Sprague-Dawley rats: Implications for preclinical models of autism spectrum disorders. PLOS ONE, 11(6), e0158150. https://doi.org/10.1371/journal.pone.0158150

Lampe, J. F., Ruchti, S., Burman, O., Würbel, H., \& Melotti, L. (2019). Play like me: Similarity in playfulness promotes social play. PLOS ONE, 14(10), e0224282. https://doi.org/10.1371/journal.pone.0224282

Lee, J., Russo, A. S., \& Parsons, R. G. (2018). Facilitation of fear learning by prior and subsequent fear conditioning. Behavioural Brain Research, 347, 61-68. https://doi.org/10.1016/j.bbr.2018.03.008

Łopuch, S., \& Popik, P. (2011). Cooperative behavior of laboratory rats (Rattus norvegicus) in an instrumental task. Journal of Comparative Psychology, 125(2), 250-253. https://doi.org/10.1037/a0021532

Manduca, A., Campolongo, P., Palmery, M., Vanderschuren, L. J. M. J., Cuomo, V., \& Trezza, V. (2014). Social play behavior, ultrasonic vocalizations and their modulation by morphine and amphetamine in Wistar and Sprague-Dawley rats. Psychopharmacology, 231(8), 1661-1673. https://doi.org/10.1007/s00213-013-33379

Martin, L. J., Tuttle, A. H., \& Mogil, J. S. (2014). The interaction between pain and social behavior in humans and rodents. In B. K. Taylor \& D. P. Finn (Eds.), Behavioral neurobiology of chronic pain (pp. 233-250). Springer. https://doi.org/10.1007/7854_2014_287

Meyza, K. Z., Bartal, I. B.-A., Monfils, M. H., Panksepp, J. B., \& Knapska, E. (2017). The roots of empathy: Through the lens of rodent models. Neuroscience \& Biobehavioral Reviews, 76, 216-234. https://doi.org/10.1016/j.neubiorev.2016.10.028

O'Connor, B. P. (1999). Simple and flexible SAS and SPSS programs for analyzing lag-sequential categorical data. Behavior Research Methods, Instruments, \& Computers, 31(4), 718-726. https://doi.org/10.3758/BF03200753

O'Keefe, D. J. (2007). Brief report: Post hoc power, observed power, a priori power, retrospective power, prospective power, achieved power: Sorting out appropriate uses of statistical power analyses. Communication Methods and Measures, 1(4), 291-299. https://doi.org/10.1080/19312450701641375

Oomen, C. A., Hvoslef-Eide, M., Heath, C. J., Mar, A. C., Horner, A. E., Bussey, T. J., \& Saksida, L. M. (2013). The touchscreen operant platform for testing working memory and pattern separation in rats and mice. Nature Protocols, 8(10), 2006-2021. https://doi.org/10.1038/nprot.2013.124

Quera, V., Bakeman, R., \& Gnisci, A. (2007). Observer agreement for event sequences: Methods and software for sequence alignment and reliability estimates. Behavior Research Methods, 39(1), 39-49. https://doi.org/10.3758/BF03192842

Sato, N., Tan, L., Tate, K., \& Okada, M. (2015). Rats demonstrate helping behavior toward a soaked conspecific. Animal Cognition, 18(5), 1039-1047. https://doi.org/10.1007/s10071-015-0872-2

Schaik, C. van, Graber, S., Schuppli, C., \& Burkart, J. (2016). The ecology of social learning in animals and its link with intelligence. The Spanish Journal of Psychology, 19, 1-12. https://doi.org/10.1017/sjp.2016.100

Seffer, D., Schwarting, R. K. W., \& Wöhr, M. (2014). Pro-social ultrasonic communication in rats: Insights from playback studies. Journal of Neuroscience Methods, 234, 73-81. https://doi.org/10.1016/j.jneumeth.2014.01.023

Sisk, C. L., \& Zehr, J. L. (2005). Pubertal hormones organize the adolescent brain and behavior. Frontiers in Neuroendocrinology, 26(3), 163-174. https://doi.org/10.1016/j.yfrne.2005.10.003

Sivaselvachandran, S., Acland, E. L., Abdallah, S., \& Martin, L. J. (2018) Behavioral and mechanistic insight into rodent empathy. Neuroscience Biobehavioral Reviews, 91,130-137. https://doi:10.1016/j.neubiorev.2016.06.007

Stryjek, R., Modlińska, K., Turlejski, K., \& Pisula, W. (2013). Circadian rhythm of outside-nest activity in wild (WWCPS), albino and pigmented laboratory rats. PLOS ONE, 8(6), e66055. https://doi.org/10.1371/journal.pone.0066055

Tomek, S. E., Stegmann, G. M., \& Olive, M. F. (2019). Effects of heroin on rat prosocial behavior. Addiction Biology, 24(4), 676-684. https://doi.org/10.1111/adb.12633 
Walker, D. M., Bell, M. R., Flores, C., Gulley, J. M., Willing, J., \& Paul, M. J. (2017). Adolescence and reward: Making sense of neural and behavioral changes amid the chaos. Journal of Neuroscience, 37(45), 1085510866. https://doi.org/10.1523/JNEUROSCI.1834-17.2017

Yamada, M., \& Sakurai, Y. (2018). An observational learning task using Barnes maze in rats. Cognitive Neurodynamics, 12(5), 519-523. https://doi.org/10.1007/s11571-018-9493-1

Yusufishaq, S., \& Rosenkranz, J. A. (2013). Post-weaning social isolation impairs observational fear conditioning. Behavioural Brain Research, 242, 142-149. https://doi.org/10.1016/j.bbr.2012.12.050

Zentall, T. R. (2016). Reciprocal altruism in rats: Why does it occur? Learning \& Behavior, 44(1), 7-8. https://doi.org/10.3758/s13420-015-0201-2 